\title{
Weak superradiance in arrays of plasmonic nanoantennas
}

\author{
Saumya Choudhary $\odot,{ }^{1,2}$ Israel De Leon,,${ }^{3,2, *}$ Sylvia Swiecicki, ${ }^{4}$ Kashif Masud Awan, ${ }^{5}$ Sebastian A. Schulz, ${ }^{6,7}$ \\ Jeremy Upham, ${ }^{7}$ M. Zahirul Alam, ${ }^{7}$ J. E. Sipe, ${ }^{4}$ and Robert W. Boyd ${ }^{1,7}$ \\ ${ }^{1}$ Institute of Optics, University of Rochester, Rochester, New York 14627, USA \\ ${ }^{2}$ School of Electrical Engineering and Computer Science, University of Ottawa, Ottawa, Ontario, Canada K1N 6N5 \\ ${ }^{3}$ School of Engineering and Sciences, Tecnológico de Monterrey, Monterrey, Nuevo León 64849, Mexico \\ ${ }^{4}$ Department of Physics, University of Toronto, Toronto, Ontario, Canada M5S 1A7 \\ ${ }^{5}$ Stewart Blusson Quantum Matter Institute, University of British Columbia, Vancouver, British Columbia, Canada V6T 1Z4 \\ ${ }^{6}$ School of Physics and Astronomy, University of Saint Andrews, Saint Andrews KY16 9SS, Scotland, United Kingdom \\ ${ }^{7}$ Department of Physics, University of Ottawa, Ottawa, Ontario, Canada K1N 6N5
}

(Received 12 March 2019; published 11 October 2019)

\begin{abstract}
A collection of $N$ emitters can exhibit an $N$-fold broadening of the radiative linewidth resulting from the development of a macroscopic dipole moment. Such a broadening of the radiative linewidth has previously been observed in systems of several nanoparticles and has often been described in terms of superradiant behavior. However, the understanding of the physics behind the observed dependence of radiative linewidth on the number of irradiated nanoparticles is far from complete. In this paper, we present theoretical and experimental results that elucidate this broadening mechanism in plasmonic systems and draw a connection with the phenomenon of Dicke superradiance. We demonstrate that, in the limit where radiative broadening dominates, the extinction linewidth of a planar array of plasmonic nanoantennas scales linearly with the number of nanoantennas contained within a circle of radius equal to the resonant optical wavelength. We explain this classical phenomenon as a weak superradiance effect, which corresponds to the case in the Dicke model where only the ground state and the first collective excited state contribute to the enhanced radiation.
\end{abstract}

DOI: 10.1103/PhysRevA.100.043814

\section{INTRODUCTION}

A collection of $N$ emitters can behave cooperatively, for example, by emitting radiation in the form of a temporally short, bright pulse [1,2]. Also associated with this cooperative radiation is the broadening of the spectrum of the collection by a factor of $N$. In the electric dipole approximation, this behavior indicates the presence of a macroscopic dipole moment in the system equal to the sum of dipole moments of the individual emitters, which results from the collective radiative interactions between the emitters [1-5]. Cooperative radiative effects were first predicted by Dicke [1], and have been demonstrated in gases [6], atomic vapors [7], Rydberg atoms [8], Bose-Einstein condensates [9], trapped ions [10], superconducting qubits [11], Mössbauer nuclei [12], and color centers in diamond [13].

Although there are quantum features of this "superradiance," the phenomenon of accelerated radiative damping of a collection of mutually coherent dipoles due to interaction with a common radiation field is essentially a classical effect. It is therefore interesting to study the cooperative interaction of dipoles in classical systems. One such system is a collection of metallic nanoparticles supporting dipolar optical resonances due to the excitation of localized surface plasmons [14]. Such dipolar modes can be modeled as classical harmonic oscillators with their light-scattering properties resembling, in many

\footnotetext{
*ideleon@tec.mx
}

ways, the radiative properties of natural dipolar emitters. Hence, plasmonic scatterers are an interesting system for the study of collective radiative behavior. Intuitively, radiative interactions in a collection of identical plasmonic nanoparticles would drive the system into developing a macroscopic dipole moment leading to the shortening of the radiative lifetime and broadening of the spectral linewidth.

Previously, coupled modes in systems of plasmonic nanoparticle dimers have been shown to exhibit a linewidth that is broader than that of each of the particles constituting the system [15-20]. The broadening of resonance linewidth has also been observed in two-dimensional collections of identical nanoparticles, such as periodic arrays of subwavelength-size split-ring resonators [21-25] and rod nanoantennas [26]. In some of these cases, the linewidth broadening has been explained either by an enhanced scattering rate due to nonradiative near-field dipole-dipole coupling [16-20,23,24] or by a retarded radiative interaction between the particles [25]. The spectral broadening in three-dimensional arrangements of nanoparticles [27-30] has also been studied, and the broadening of the transmittance spectrum of a periodic threedimensional arrangement of plasmonic nanowires has been explained by a far-field coupling between the wires [27]. Furthermore, cooperative radiative behavior has been studied in various arrangements of plasmonic nanospheres, such as gold nanospheres trapped in a linear array by a tightly focused laser beam [28], a plastic bead coated with gold nanospheres [29], and random aggregates of gold and silver nanospheres [30]. In each of these studies, the linewidth of scattered light scales 
linearly with the number of irradiated nanospheres, which has been phenomenologically associated with a corresponding increase in the radiative decay rate [28-30].

Despite the various observations of radiative spectral broadening in plasmonic systems, a detailed analysis of the emergence of the radiative linewidth dependence on the number of irradiated plasmonic nanoparticles has not yet been presented. In this paper, we study the collective behavior in arrays of identical plasmonic nanoparticles through experiment, finite-difference time-domain (FDTD) simulations, and an analytical model. We show that the radiative linewidth of a planar array of nanoantennas is directly proportional to the effective number of nanoantennas, $N_{\text {eff }}$, contained within a circle of radius equal to the resonant optical wavelength, $\lambda_{0}$. In addition, we show that in the dipole approximation this linewidth dependence is due to the enhancement of the radiation reaction field of each nanoantenna by that of its neighbors in the array, and is, therefore, a cooperative effect. Finally, we draw a connection between the "plasmonic superradiance" observed in our system and Dicke superradiance and argue that the plasmonic system is analogous to a system of weakly excited emitters in the Dicke model.

\section{EXPERIMENT AND NUMERICAL ANALYSIS}

A schematic representation of the structure under investigation is shown in Fig. 1(a). It consists of a planar square array of bar-shaped gold nanoantennas with subwavelength dimensions, placed on a 1-mm-thick BK7 (or float) glass substrate. The background medium is made symmetric by covering the array by a medium with the same permittivity as the substrate, which in practice entails coating the fabricated sample with index-matched oil, followed by a float glass cover slip. The arrays are excited by normally incident broadband light, and we study the dependence of the linewidth of scattered light from the array as a function of the number density of nanoantennas or, equivalently, of the array's lattice constant, $a$. The dimensions of the nanoantenna are chosen such
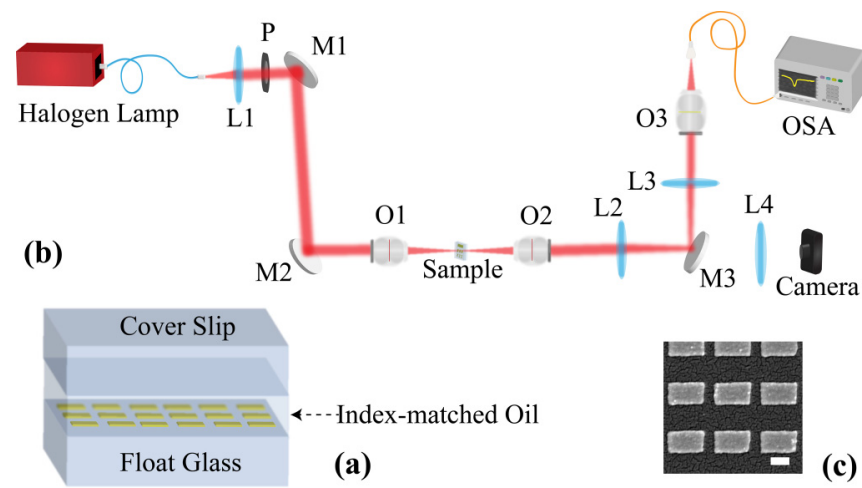

(c)

FIG. 1. (a) Schematic of the experimental setup. The labels on the schematic are as follows: L1, collimating lens; $\mathrm{P}$, linear polarizer; $\mathrm{O} 1$ and $\mathrm{O} 2$, microscope objectives $(\mathrm{O} 1, \times 10$ and $\mathrm{NA}=0.25 ; \mathrm{O} 2$, $\times 4$ and NA $=0.1$ ); L2-L4, imaging lenses; OSA, optical spectrum analyzer; M1 and M2, folding mirrors; M3, flip mirror. (b) A crosssection view of the sample showing a nanoantenna array (gold bars). (c) Scanning electron micrograph of one of the fabricated arrays. The solid white bar in the lower right corner is $100 \mathrm{~nm}$ long for scaling. that the dominant damping mechanism is radiative, thereby allowing the observation of variations in radiative linewidth simply by measuring the transmission spectrum of the array. To this end, the scattering and absorption cross sections of the nanoantenna are calculated via FDTD simulations. For the selected dimensions of the nanoantenna (185 nm long, 105 $\mathrm{nm}$ wide, and $20 \mathrm{~nm}$ thick; these are also roughly the average dimensions of the nanoantennas in the fabricated arrays), the resonant scattering, which occurs at a wavelength (frequency) of $1181 \mathrm{~nm}(254 \mathrm{THz})$, is more than three times as strong as the absorption (see Fig. 4 in Appendix A).

We have fabricated 11 squared arrays of nanoantennas, with lattice constants ranging from 250 to $500 \mathrm{~nm}$ in steps of $25 \mathrm{~nm}$, using electron-beam lithography in a Raith Pioneer 30-kV electron-beam system to form a patterned resist on a 1$\mathrm{mm}$-thick float glass substrate. A gold film of thickness $20 \mathrm{~nm}$ is then deposited, and the resist is removed by the process of liftoff, leaving behind the patterned gold on the substrate [31]. Appendix D has a detailed description of the fabrication procedure. The size of each array is $200 \times 200 \mu \mathrm{m}$. The scanning electron micrograph in Fig. 1(c) shows a detail of one of the fabricated arrays.

The transmission spectra of the fabricated arrays are measured by transmission spectroscopy using the setup depicted schematically in Fig. 1(b). The normalized average transmission spectrum $T$ of each array was obtained by taking the ratio of the transmission spectrum of each array to the transmission spectrum of the glass-oil assembly, with each spectrum averaged over three measurements. As the response of a single nanoantenna to an incident field can be described by contributions from the excited multipoles [32], the collective response of nanoantennas in each array also consists of multipolar contributions. However, as evidenced in the crosssection spectra and the near-field profile of an isolated nanoantenna (see Fig. 4 in Appendix A), for the range of frequencies under consideration the dipole mode of the nanoantenna is dominant. Hence, the radiative decay rate of the collective dipolar mode of each array is obtained from the full width at half maximum (FWHM) linewidth of the corresponding extinction spectrum $(1-T)$.

In Figs. 2(a) and 2(b) we compare the extinction spectra of the nanoantenna arrays of different lattice constants obtained from FDTD simulations and from the experiment, respectively. For the FDTD simulation of the periodic nanoantenna array, we have considered a single unit cell of the array with periodic boundary conditions at the transverse boundaries. As a consequence, the uniformity of dimensions of the nanoantennas in each array was implicitly assumed. On the other hand, for the fabricated arrays, there are unavoidable inhomogeneities in the dimensions of the nanoantenna, which contribute to inhomogeneous broadening. In addition, in the experiment the exciting field could have some obliquely incident components despite the use of objectives with low numerical apertures. These factors could explain the slight differences between the simulated and the measured spectra. However, both measured and simulated spectra show similar broadening of the extinction linewidth with decreasing array lattice constant, which is consistent with the expectation that increasing the number of dipoles within an optical wavelength broadens their radiative linewidth. 


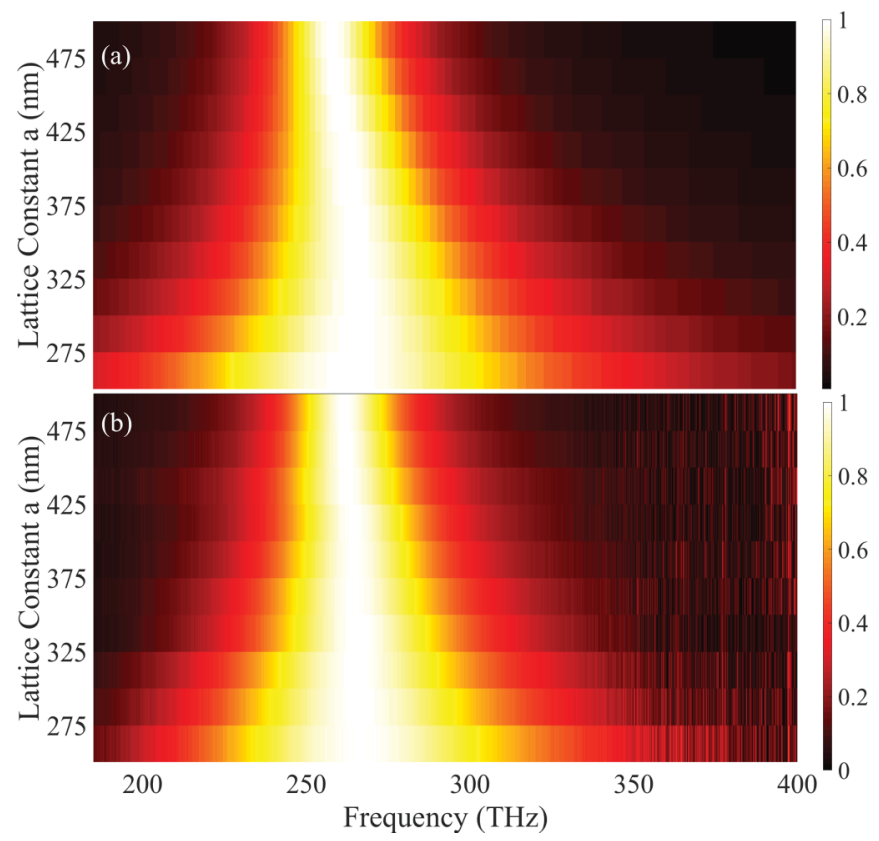

FIG. 2. Extinction spectra of nanoantenna arrays of different lattice constants obtained from (a) FDTD simulations and from (b) the experiment.

\section{ANALYTICAL MODEL}

In order to gain physical insight into the relation between the radiative linewidth and the lattice constant, we introduce an analytic model to describe the scattered field from an array of nanoantennas. We approximate each nanoantenna as a point dipole of electric dipole moment $\boldsymbol{p}$, and begin by introducing the polarizability tensor $\overleftrightarrow{\alpha_{0}}$ in the electrostatic limit. For ease of analytical calculations, each nanoantenna is modeled as ellipsoids with identical volume and aspect ratios. We assume our coordinate system to be aligned along the principal axes of the ellipsoid [see inset in Fig. 5(a)]. The polarizability tensor $\overleftrightarrow{\alpha_{0}}$ is then represented by a diagonal matrix, and its elements are proportional to the volume of the ellipsoid. The diagonal elements, $\alpha_{0, \text { ii }}$ for $i=\{x, y, z\}$, describe the response of the electric dipole excited along the corresponding axis, and depend on the exact shape and permittivities of the nanoantenna as well as the surrounding medium; the form for a gold ellipsoid embedded in glass is detailed in Appendix B. The implicit dependence of $\overleftrightarrow{\boldsymbol{\alpha}_{0}}$ on the frequency $\omega$ includes the effects of material dispersion of both the nanoantenna and the surrounding medium. The resonance frequency $\omega_{0}$ of the dipole is obtained from the condition $\operatorname{Re}\left[\alpha_{0, \text { ii }}^{-1}\left(\omega_{0}\right)\right]=0$. In addition, in the electrostatic limit the damping of the dipole is purely nonradiative, and the FWHM linewidth of the corresponding extinction spectrum [Eq. (B5)] depends only on the material losses (characterized by $\left.\operatorname{Im}\left[\alpha_{0, \mathrm{ii}}^{-1}\right]\right)$.

We first consider an isolated ellipsoidal nanoantenna, excited by an incident field of frequency $\omega$ that has a value $\boldsymbol{E}_{\text {inc }}$ at the center of the nanoantenna. There are two features in the response that arise when we go beyond electrostatics. First, in the neighborhood of the nanoantenna there is a field contribution from the nanoantenna itself that is out of phase with its dipole moment, but proportional to it, and is responsible for the radiation reaction. Second, if the volume $V$ of the nanoantenna is not significantly smaller than $\lambda_{0}^{3}$, there is a part of the field from the nanoantenna which varies across the nanoantenna due to retardation. The effects due to these two features of the response on the electric dipole moment $\boldsymbol{p}$ of the nanoantenna induced can still be captured within a point dipole model, but with the replacement of $\boldsymbol{E}_{\text {inc }}$ by a modified field $\boldsymbol{E}_{\text {mod }}$. That is, we can write

$$
\boldsymbol{p}=\epsilon_{0} n^{2} \overleftrightarrow{\boldsymbol{\alpha}_{0}} \cdot \boldsymbol{E}_{\mathrm{mod}},
$$

where

$$
\boldsymbol{E}_{\text {mod }}=\boldsymbol{E}_{\mathrm{inc}}+\frac{1}{4 \pi \epsilon_{0}} \frac{2}{3} i n \tilde{\omega}^{3} \boldsymbol{p}+\overleftrightarrow{\boldsymbol{\beta}_{0}} \cdot \frac{1}{\epsilon_{0} n^{2}} \boldsymbol{p} .
$$

Here $n$ is the refractive index of the surrounding medium (here BK7 glass), and $\tilde{\omega}=\omega / c$. In (2), the second term on the right is the radiation reaction field from the nanoantenna itself, and the dyadic $\overleftrightarrow{\boldsymbol{\beta}_{0}}$, sometimes referred to as the "dynamic depolarization term," depends on the size and shape of the nanoantenna, is purely real, and is associated with the variation of the full electric field over the nanoantenna due to retardation $[33,34]$. In Appendix B we give the expression for $\overleftrightarrow{\boldsymbol{\beta}_{0}}$ for our ellipsoids.

The aforementioned electrodynamic contributions to the response of the nanoantenna to the incident field can alternately be captured by introducing a new polarizability $\overleftrightarrow{\alpha}$ that relates $\boldsymbol{p}$ to the incident field $\boldsymbol{E}_{\text {inc }}$ as follows [35]:

$$
\boldsymbol{p}=\epsilon_{0} n^{2} \stackrel{\leftrightarrow}{\alpha} \cdot \boldsymbol{E}_{\mathrm{inc}}
$$

where $\overleftrightarrow{\alpha}$ is also a diagonal matrix in the chosen coordinate system. From (1) and (3), and using the property that the diagonal matrices $\overleftrightarrow{\alpha_{0}}$ and $\overleftrightarrow{\alpha}$, and their inverses, all commute with each other, we then write

$$
\overleftrightarrow{\boldsymbol{\alpha}}^{-1} \cdot \boldsymbol{E}_{\mathrm{mod}}=\overleftrightarrow{\boldsymbol{\alpha}}_{0}^{-1} \cdot \boldsymbol{E}_{\mathrm{inc}}
$$

Then, using (2) and (3) in (4), we can write after some simplification

$$
\overleftrightarrow{\boldsymbol{\alpha}}^{-1}={\overleftrightarrow{\boldsymbol{\alpha}_{0}}}^{-1}-\frac{i}{6 \pi}(n \tilde{\omega})^{3} \overleftrightarrow{\boldsymbol{U}}-\overleftrightarrow{\boldsymbol{\beta}_{0}},
$$

where $\overleftrightarrow{\boldsymbol{U}}$ is the unit dyadic. Recall that in general $\operatorname{Im}\left[{\overleftrightarrow{\boldsymbol{\alpha}_{0}}}^{-1}\right]$ describes nonradiative losses due to electron collisions within the nanoantenna, and free-carrier absorption and interband transitions in the gold [14]. While the losses due to electron scattering are not relevant for the dimensions of the nanoantennas considered here, the losses due to bulk free-carrier absorption and interband transitions (the latter being negligible for our wavelengths) are captured by using the frequencydependent dielectric constant of the gold. In addition to these nonradiative losses, we can identify the radiation reaction term in (5) as describing radiative losses due to the scattering of the electromagnetic field from the nanoantenna. Both the radiative and nonradiative losses will broaden its extinction spectrum. The dynamic depolarization term, on the other 
hand, leads to a shift in the resonance frequency from its value in the electrostatic limit. We note that similar expressions for the dipolar polarizability of spheres and spheroids have been calculated previously from the modified long-wavelength approximation [34] or from Padé approximations of Mie scattering coefficients [36].

We now consider a square lattice of these dipoles lying in the $x y$ plane. For a normally incident field we find that all the dipole moments will be identical, with

$$
\boldsymbol{p}=\epsilon_{0} n^{2} \overleftrightarrow{\boldsymbol{\alpha}_{0}} \cdot \boldsymbol{E}_{\mathrm{tot}},
$$

where $\boldsymbol{E}_{\text {tot }}$ includes the field $\boldsymbol{E}_{\text {mod }}$, given by (2), along with the field from the remaining nanoantennas in the array:

$$
\boldsymbol{E}_{\mathrm{tot}}=\boldsymbol{E}_{\mathrm{inc}}+\frac{1}{4 \pi \epsilon_{0}} \frac{2}{3} i n \tilde{\omega}^{3} \boldsymbol{p}+\left(\stackrel{\leftrightarrow}{\boldsymbol{\beta}_{0}}+\overleftrightarrow{\boldsymbol{\beta}}\right) \cdot \frac{1}{\epsilon_{0} n^{2}} \boldsymbol{p}
$$

The dyadic $\stackrel{\leftrightarrow}{\boldsymbol{\beta}}$ is the "dynamic interaction constant" of the array [37], and is given by

$$
\overleftrightarrow{\boldsymbol{\beta}}=\sum_{\boldsymbol{R} \neq 0} \stackrel{\leftrightarrow}{\boldsymbol{G}}(-\boldsymbol{R})
$$

with $\stackrel{\leftrightarrow}{\boldsymbol{G}}$ being the periodic Green dyadic [38], such that $\overleftrightarrow{\boldsymbol{G}}$ $(-\boldsymbol{R}) \cdot \boldsymbol{p}$ represents the electric field at the origin due to a dipole moment $\boldsymbol{p}$ at a lattice site $\boldsymbol{R}$.

Similar to the strategy used in treating an isolated nanoantenna, we can redefine the polarizability of each nanoantenna in the array with respect to the incident field as

$$
\boldsymbol{p}=\epsilon_{0} n^{2} \stackrel{\leftrightarrow}{\boldsymbol{\alpha}}_{\mathrm{eff}} \cdot \boldsymbol{E}_{\mathrm{inc}},
$$

and we find that the effective polarizability $\overleftrightarrow{\alpha}_{\text {eff }}$ of a nanoantenna in a square array is given by

$$
\overleftrightarrow{\boldsymbol{\alpha}}_{\mathrm{eff}}^{-1}={\overleftrightarrow{\boldsymbol{\alpha}_{0}}}^{-1}-\frac{i}{6 \pi}(n \tilde{\omega})^{3} \overleftrightarrow{\boldsymbol{U}}-\left(\overleftrightarrow{\boldsymbol{\beta}_{0}}+\overleftrightarrow{\boldsymbol{\beta}}\right)
$$

To calculate the the poorly convergent $\overleftrightarrow{\boldsymbol{\beta}}$, we use the Poisson summation method followed by singularity cancellation, as discussed in [37,38]. Assuming the incident electric field to be polarized along the major axis of the nanoantenna [aligned with the $x$ axis, as shown in Fig. 5(a)], only the dipole along the $x$ axis is excited. Hence, the response of the array is given by the diagonal component of the effective polarizability $\left[\alpha_{\text {eff }}\right]_{x x}$. For our specific case (square lattices with subwavelength lattice constant $a$ excited by normally incident light), the exact analytic result for the imaginary part of the dynamic interaction constant $\beta_{x x}$, also known as the "lattice sum," can be obtained from Eqs. (76) and (110) in Swiecicki and Sipe [38], and is given by

$$
\operatorname{Im}\left[\beta_{x x}\right]=-\frac{1}{6 \pi}(n \tilde{\omega})^{3}+\frac{n \tilde{\omega}}{2 a^{2}} .
$$

In contrast, $\operatorname{Re}\left[\beta_{x x}\right]$ does not have a closed-form expression, and is actually an infinite series [37]. We numerically evaluate the series in our calculations by including as many terms as required until convergence is achieved for the extinction spectrum.
From (11) and (10), now we can write $\left[\alpha_{\text {eff }}\right]_{x x}$, while dropping the suffix $\mathrm{xx}$, as

$$
\alpha_{\text {eff }}^{-1}=\alpha_{0}^{-1}-\frac{i}{6 \pi}(n \tilde{\omega})^{3} N_{\text {eff }}-\left(\beta_{0}+\operatorname{Re}[\beta]\right),
$$

where $N_{\text {eff }}=3 \lambda^{2} / 4 \pi n^{2} a^{2}$ is the effective number of dipoles enclosed within a circle of radius equal to the wavelength in the background medium, $\lambda / n$. We note that the effective depolarization term of the dipole in the array, $\operatorname{Re}\left[\alpha_{\text {eff }}^{-1}\right]$, is enhanced by the "collective retardation" term, $\operatorname{Re}[\beta]$, which is responsible for the shift in the resonance frequency (given by $\operatorname{Re}\left[\alpha_{\text {eff }}^{-1}\left(\omega_{0}\right)\right]=0$, or the maxima of the extinction spectra) for different arrays observed in Figs. 2(a) and 2(b). We also note from (12) that there are no collective contributions to the nonradiative losses due to absorption, which are once again represented by the term $\operatorname{Im}\left[\alpha_{0}^{-1}\right]$. On the other hand, the radiative damping term has the form of the radiationreaction term of $N_{\text {eff }}$ dipoles. This collectively enhanced radiative damping is responsible for the observed linewidth broadening in Figs. 2(a) and 2(b). Finally, the transmittance $T$ and reflectance $R$ spectra of the array depend on $\alpha_{\text {eff }}(\omega)$ as follows [39]:

$$
\begin{aligned}
& R=|r|^{2}=\frac{(n \tilde{\omega})^{2}\left|\alpha_{\mathrm{eff}}\right|^{2}}{4 a^{4}}, \\
& T=|t|^{2}=1+R-\frac{n \tilde{\omega}}{a^{2}} \operatorname{Im}\left[\alpha_{\mathrm{eff}}\right] .
\end{aligned}
$$

Since the effective number of dipoles $N_{\text {eff }}$ within a wavelength-sized area is proportional to $a^{-2}$, in the regime where scattering dominates absorption, the FWHM linewidth of the extinction spectrum $(1-T)$ should decrease according to $a^{-2}$ as the lattice constant of the planar array is increased.

The extinction spectra of the equivalent ellipsoid arrays for various lattice constants are analytically calculated from Eqs. (13). The dimensions of the ellipsoid (semiprincipal axes of $a_{e}=112 \mathrm{~nm}, b_{e}=63.85 \mathrm{~nm}$, and $c_{e}=12.25 \mathrm{~nm}$ ) have been chosen so as to have the closest agreement between the extinction spectrum of the single nanoantenna obtained through FDTD simulations and that of the gold ellipsoid obtained analytically for light polarized along their long axes. We used the Johnson and Christy values for the permittivity of gold [40] for both FDTD simulations and our analytical calculations. See Appendix B for the details of the calculation of the equivalent ellipsoid parameters. Figure 6 in Appendix C shows the analytically calculated extinction spectra of the ellipsoid arrays with lattice constants ranging from 250 to 500 $\mathrm{nm}$ in steps of $25 \mathrm{~nm}$. Comparing Figs. 2(a) and 2(b) with Fig. 6, we find a good qualitative agreement between our analytical model, FDTD simulations, and experimental results.

\section{RESULTS AND DISCUSSION}

In Fig. 3 we show the lattice constant dependence of the measured, FDTD simulated, and analytically calculated FWHM linewidths of the extinction spectra; the last of these follows from using Eqs. (6)-(13). For the datasets obtained from FDTD simulations (red triangles) and the experiment (blue circles), only the linewidths of the spectra shown in Figs. 2(a) and 2(b), respectively, are plotted in Fig. 3. For the analytical dataset, the extinction spectra of equivalent 


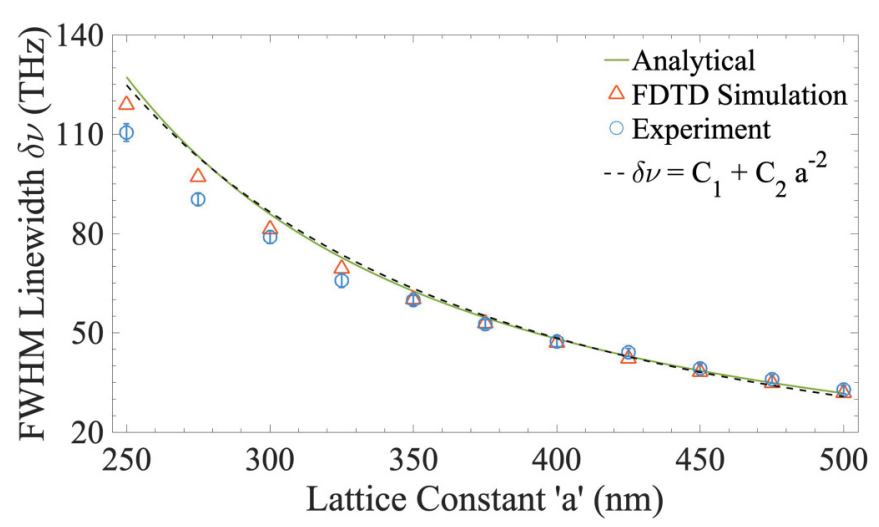

FIG. 3. FWHM linewidths vs lattice constant of extinction spectra obtained from experiment (circles, blue), simulations (triangles, red), and analytical calculations (solid, green). The black dashed curve represents the fitted curve $\delta v=C_{1}+C_{2} a^{-2}$ to the analytical data, where $C_{1}=-0.1694 \mathrm{THz}$ and $C_{2}=7.73 \times 10^{6} \mathrm{THz} \mathrm{nm}{ }^{2}$.

ellipsoid arrays were calculated for arrays with lattice constants varying from 250 to $500 \mathrm{~nm}$ in steps of $1 \mathrm{~nm}$. The FWHM linewidths of these analytically calculated spectra are plotted in Fig. 3 as a continuous (green) line. The error bars on the experimental data indicate the experimental error in determining the FWHM from the optical spectrum analyzer readout as a consequence of noise in its detector.

A simple expression for the dependence of the FWHM on the lattice constant is difficult to extract even for the analytic model, because the resonant frequency itself depends on the lattice constant $a$. However, for the form of the polarizability $\alpha_{0}$ adopted (see Appendix B), and recognizing that the shift of the resonant frequency due to the real part of $\beta$ and the linewidth itself are reasonably small compared to the resonant frequency $\omega_{0}$, a linewidth dependence of the form $\delta v=$ $C_{1}+C_{2} a^{-2}$ can be expected, where $C_{1}$ captures the effects of absorption and $C_{2} a^{-2}$ captures the effects of radiative broadening. The constants $C_{1}$ and $C_{2}$ are obtained by fitting the aforementioned curve to the analytical dataset. The fitted curve $\left(C_{1}=-0.1694 \mathrm{THz}\right.$ and $\left.C_{2}=7.73 \times 10^{6} \mathrm{THz} \mathrm{nm}^{2}\right)$ is shown as a dashed black line in Fig. 3, and we see a good agreement between the analytical dataset and the fitted curve. Comparing the numerical values of $C_{1}$ and $C_{2}$, we note that the term $C_{2} a^{-2}$ has a significantly larger contribution to $\delta v$, thereby indicating that the radiative broadening effects dominate over those of absorption.

For lattice constants larger than about $375 \mathrm{~nm}$ there is an excellent quantitative fit between experiment, FDTD simulation, and the analytic model. At smaller lattice constants we see that the results of the FDTD simulations differ from those of the analytic model. This may be in part due to the fact that the FDTD simulations were done for the actual array of rod-shaped nanoparticles, which includes contributions from all multipolar excitations of the nanoparticle as well as their interactions with the other nanoparticles in the array. In contrast, the analytic model relies on a fit to the response of an isolated nanoparticle with ellipsoidal shape, and the use of a point dipole approximation for calculating the interaction between the nanoparticles in the array. The validity of these features of the analytic model becomes questionable for very small lattice constants, giving a possible reason for the difference between the FDTD simulations and the analytic model for small values of $a$. We also see that at small lattice constants the experimental results differ from the results of the FDTD simulations, which may be due to inhomogeneities in the properties of the fabricated nanorods. The inevitable fabrication imperfections would contribute to some inhomogeneous broadening in the experimental case, which is not an issue in the analytical model or FDTD simulations due to our assumption of all the nanoantennas being identical. The effect of these inhomogeneities in the fabricated nanorods can be expected to have a larger effect at smaller lattice constants where the response of a given nanorod is more sensitive to the details of the near field from its neighbors, and thereby could also contribute to the mismatch between the experiment and the simulations for smaller lattice constants.

Nonetheless, within a reasonable margin of error our results indicate that the observed $N_{\text {eff }}$-fold radiative linewidth broadening in the arrays of nanoantennas is a collective effect. We have also shown through the closed-form expression of the imaginary part of the effective polarizability of each nanoantenna within the array that the observed $N_{\text {eff }}$-dependent linewidth scaling is because the radiation reaction of the array is proportional to $N_{\text {eff }}$ (12). In addition, since we have explicitly designed our nanoantennas to have a much larger scattering cross section than the absorption cross section, we conclude that the observed linewidth broadening is due to the interaction of the plasmonic scatterers with the common radiation field.

The scaling of the linewidth with the number density of dipoles indicates a connection with Dicke superradiance, where the interaction with the common radiation field is also the important physics. Dicke [1] dealt with two-level systems, while our plasmonic scatterers can be well described by a harmonic oscillator model; in general one would only expect a correspondence in the weak excitation limit. With that in mind we look at the first excited state in the Dicke model, which consists of a single excitation "shared" among the $N$ two-level systems. The total dipole matrix element between this lowest excited state and the ground state is a factor of $\sqrt{N}$ larger than the dipole matrix element between the excited state and the ground state of a single two-level system, and so if the $N$ two-level systems are confined within a wavelength of light the emission rate from this first excited state will be a factor of $N$ larger than for a single excited two-level system, leading to a linewidth that is a factor of $N$ times that for a single excited two-level system. This can be called "weak superradiance," to distinguish it from the behavior of other excited states in the Dicke model that have much enhanced decay rates. In a scattering problem in the semiclassical approximation, where the radiation field is treated classically, it is the dipole moment between the ground state and the first excited state of the Dicke model that is relevant for weak enough incident fields. There again one finds a linewidth that is $N$ times as large as would be seen if only a single two-level system were considered [35,41-45]. Now if only the ground state and the first excited state are considered, then the Dicke model is essentially equivalent to a set of harmonic oscillators, since for the latter system the first excited state also consists of a single shared excitation, here shared between the harmonic 
oscillators. Thus we see that the behavior considered here for plasmonic oscillators mimics the corresponding behavior of two-level systems. So, whether considering plasmonic oscillators or two-level systems under weak excitation, for planar arrays of effective atoms the relevant $N$ is the number of those atoms within a square wavelength of light.

\section{CONCLUSION}

To summarize, here we have shown that the linewidth of light scattered by a planar array of plasmonic nanoantennas scales linearly with the effective number of nanoantennas contained within an area equal to the square of the resonant optical wavelength. Through an analytical model, we have shown that this is a collective effect resulting from the enhancement of the radiation reaction of a particular nanoantenna by the radiation reaction of all the other nanoantennas in the array, which in turns leads to an $N_{\text {eff-fold enhance- }}$ ment of the radiative linewidth. In an effort to elucidate the relationship between the collective radiative behavior of plasmonic scatterers and the behavior of Dicke superradiance, we have also discussed the different radiative behaviors of plasmonic scatterers and dipolar emitters. We have argued that the collective radiative behavior of a system of plasmonic scatterers and a corresponding system of weakly excited emitters (wherein only one emitter is initially excited on an average) are analogous. Accordingly, we have termed this effect "weak superradiance."

It should be noted that the analytical method used here to calculate the effective dipolar polarizability can be extended to include electric quadrupole and magnetic dipole contributions to the array response [38]. The inclusion of these multipolar contributions should provide insight not only into the slight difference between the results from the analytical model and FDTD simulations that we observed for smaller lattice constants, but also into other interesting phenomena such as Fano resonances and directional scattering from metasurfaces $[46,47]$. Further, the model could also be extended to study a wide range of metasurfaces, including arrays of split-ring resonators and dielectric nanoparticles [47,48], as well as multilayer metasurfaces $[27,49]$.

\section{ACKNOWLEDGMENTS}

The authors acknowledge financial support from the Canada Excellence Research Chairs (CERC) Program, the Natural Sciences and Engineering Research Council of Canada (NSERC), and the US Office of Naval Research (Grants No. N00014-17-1-2443 and No. N00014-17-S-B001).

\section{APPENDIX A: NANOROD CROSS SECTIONS}

Figure 4 shows the scattering, absorption, and extinction cross sections of an isolated nanorod calculated through FDTD simulations. The resonant scattering (at $254 \mathrm{THz}$ ) is more than three times as strong as the absorption. The electric-field profile at the resonance (the inset) indicates that the resonant mode is dipolar. The arrow represents the polarization of the incident field.

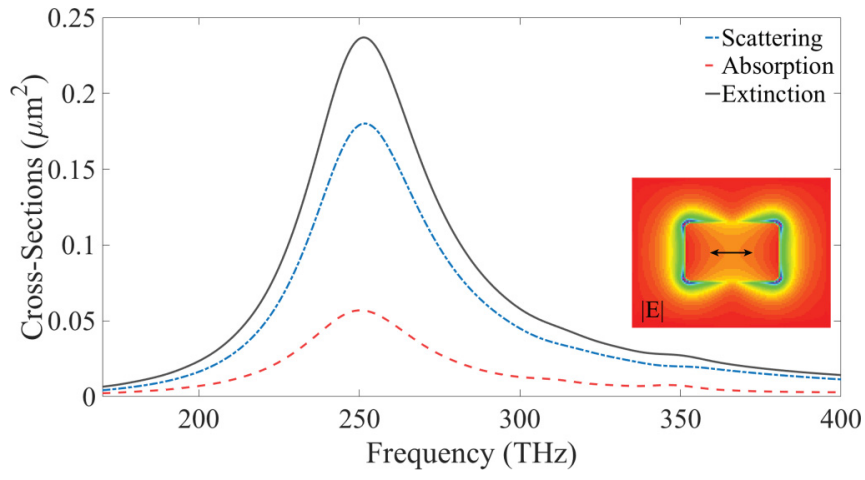

FIG. 4. Scattering (dot-dashed, blue), absorption (dashed, red), and extinction (solid, gray) cross-section spectra of a single isolated nanorod. The inset shows the electric-field mode profile at resonance.

\section{APPENDIX B: EQUIVALENT ELLIPSOID PARAMETER CALCULATION}

From the electrostatic model for dipole polarizability of an ellipsoid of semiaxes lengths $a_{e}, b_{e}$, and $c_{e}$, we have the following expression [50]:

$$
\alpha_{0, i i}=V\left(L_{i}+\frac{\epsilon}{\epsilon_{1}-\epsilon}\right)^{-1},
$$

where $i=x, y, z ; V=4 \pi a_{e} b_{e} c_{e} / 3$ is the volume of the ellipsoid; $\epsilon_{1}$ and $\epsilon$ are the material permittivity of the ellipsoid (gold) and of the surrounding medium (BK7 glass), respectively. $L_{i}$ is the shape parameter (or the depolarization factor) given by

$$
L_{i}=\frac{a_{e} b_{e} c_{e}}{2} \int_{0}^{\infty} \frac{d q}{f(q)\left(q+d_{i}^{2}\right)},
$$

with $d_{i}=\left\{a_{e}, b_{e}, c_{e}\right\} \quad$ for $\quad i=\{x, y, z\}, \quad$ and $\quad f(q)=$ $\sqrt{\left(q+a_{e}^{2}\right)\left(q+b_{e}^{2}\right)\left(q+c_{e}^{2}\right)}$. The optical constants of gold (for $\epsilon_{1}$ ) were taken from Johnson and Christy [40], and the permittivity of the BK7 glass $\left(\epsilon_{m}\right)$ was taken from [51]. The only damping mechanism in this limit arises from the material response, which is independent of the dimensions of the ellipsoid. For ellipsoids of volumes as large as the nanorod (185 nm long, $105 \mathrm{~nm}$ wide, and $20 \mathrm{~nm}$ thick), radiative damping becomes a significant contribution to the linewidth, along with the nonradiative damping due to the material response. In addition, retardation effects come into play, which lead to a shift in the resonance frequency. These effects are not accounted for in the electrostatic polarizability model. Kuwata et al. [52] provide an empirical model for an arbitrary shaped nanoparticle that takes into account retardation as well as the radiation reaction by approximating the total polarizability as

$$
\begin{gathered}
\alpha_{i i}^{-1} \approx \alpha_{0, i i}^{-1}-i \frac{(n \tilde{\omega})^{3}}{6 \pi}-\beta_{0, i i}, \\
\beta_{0, i i}=-\frac{A\left(L_{i}\right)}{V}\left(n \tilde{\omega} d_{i}\right)^{2}-\frac{B\left(L_{i}\right)}{V}\left(n \tilde{\omega} d_{i}\right)^{4},
\end{gathered}
$$

where $\tilde{\omega}=\omega / c$ and $n$ is the refractive index of the surrounding medium. The second term on the right-hand side of Eq. (3) accounts for the radiation reaction, while the $\beta_{0, i i}$ term is an empirical term that accounts for retardation. $A\left(L_{i}\right)$ 
and $B\left(L_{i}\right)$ are polynomials of the shape parameter $L_{i}$. The specific form of the polynomials depends on the geometry of the nanoparticles under consideration and does not depend on the material being considered. The resonance condition is met when the real part of the right-hand side of Eq. (3) vanishes. In addition, from (B1) and (B3), we see that a larger volume of the ellipsoid leads to larger radiative damping (and hence larger spectral linewidth), as well as a redshift in the resonance [34,53].

The cross sections of the ellipsoid are given by (assuming $i=x$ and dropping the suffixes)

$$
\begin{aligned}
C_{\mathrm{ext}} & =n \tilde{\omega} \operatorname{Im}[\alpha], \\
C_{\mathrm{scat}} & =\frac{(n \tilde{\omega})^{4}}{6 \pi}|\alpha|^{2}, \\
C_{\mathrm{abs}} & =C_{\mathrm{ext}}-C_{\mathrm{scat}} .
\end{aligned}
$$

Kuwata et al. [52] provide the polynomials $A(L)$ and $B(L)$ for spheroids of aspect ratios larger than 3.8. However, for the aspect ratios of the nanorods that we have considered, the polynomials given in [52] do not yield correct results. In order to obtain the correct form of the polynomials specific to our case, we compare the cross-section spectra of ellipsoids of different dimensions simulated in FDTD with the cross-section spectra corresponding to ellipsoid polarizability given by (B3)

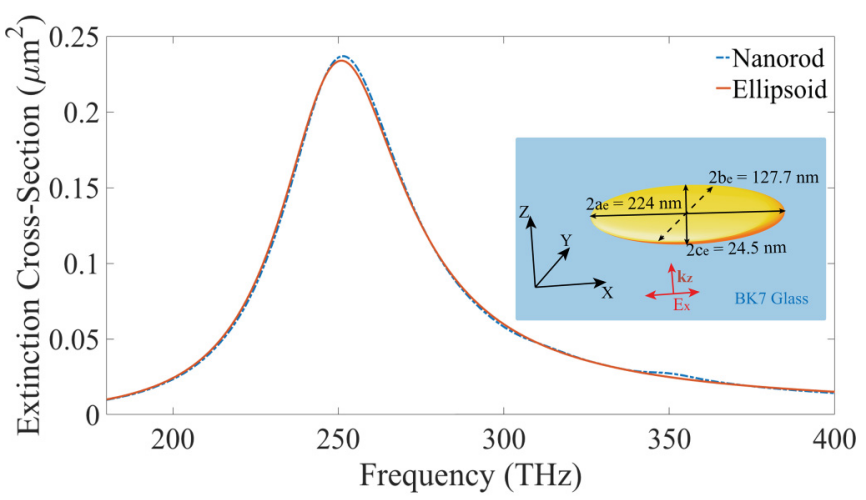

(a)

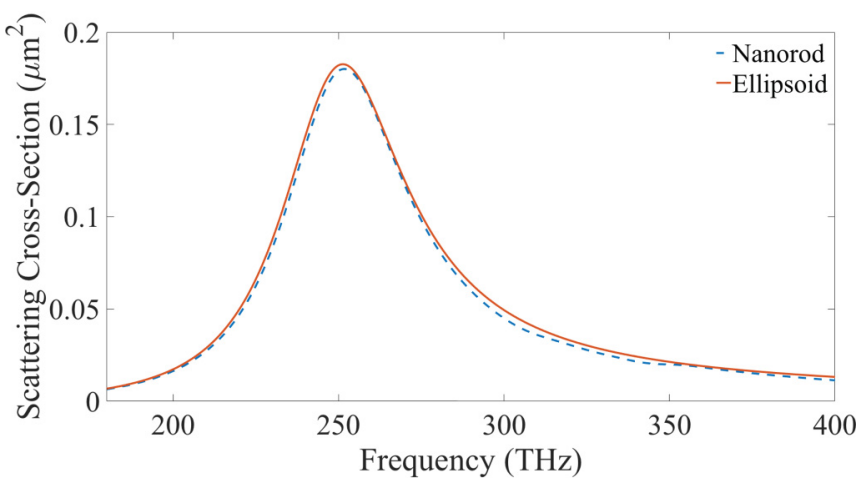

(b)

FIG. 5. (a) Extinction and (b) scattering cross section vs frequency for the nanorod (blue, from FDTD simulations) and the ellipsoid (red, from the analytical dipole polarizability model). In (a), the inset shows the ellipsoid dimensions and excitation geometry used for analytical calculations.

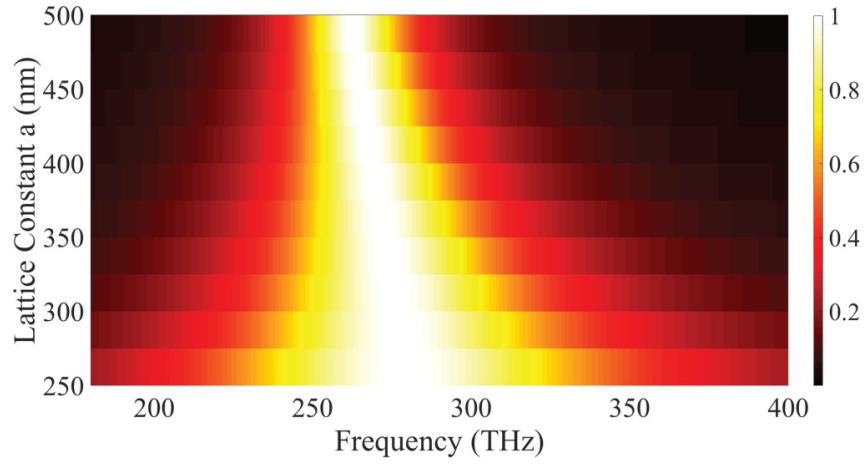

FIG. 6. Analytically calculated extinction spectra of the ellipsoid arrays of different lattice constants.

while assuming different forms of the polynomials $A(L)$ and $B(L)$. The closest agreement between the FDTD simulations and the analytical model was found for $A(L)=-0.4915 L-$ $1.046 L^{2}+0.8481 L^{3}$ and dropping the term associated with the polynomial $B(L)$.

The dimensions of the ellipsoid with scattering and extinction spectra [obtained from Eqs. (B3), (B5), and (B6)] in closest agreement with the corresponding spectra of an isolated nanorod are $a_{e}=112 \mathrm{~nm}, b_{e}=63.85 \mathrm{~nm}$, and $c_{e}=$ $12.25 \mathrm{~nm}$. Figure 5(a) [Fig. 5(b)] compares the extinction (scattering) cross-section spectrum of the nanorod, obtained from FDTD simulations, with the corresponding analytically calculated spectrum of the ellipsoid of aforementioned dimensions.

\section{APPENDIX C: ANALYTICAL SPECTRA}

Figure 6 shows the analytically calculated extinction spectra of the ellipsoid arrays of different lattice constants obtained by substituting the calculated polarizability of the ellipsoid from Eq. (B3) into Eqs. (13) given in the main text. As stated in the main text, the analytically calculated spectra are very similar to the simulated and measured spectra shown in Figs. 2(a) and 2(b), respectively. From Fig. 5(b), we see that the scattering linewidth of the ellipsoid is slightly broader (by approximately $1 \mathrm{THz}$ ). This implies a slightly larger radiative damping of the ellipsoid compared to the nanorod, and hence a slightly larger radiative linewidth for decreasing (increasing) lattice constant $\left(N_{\text {eff }}\right)$, as predicted by Eq. (12) in the main text. To ensure a further agreement between the analytical calculations and the experiment, one can extract the dipole polarizability of the nanorod itself and substitute in Eqs. (12) and (13) of the main text to calculate the extinction spectra. Nevertheless, for all practical purposes, the present analysis provides sufficient evidence that the enhancement in linewidth is due to a corresponding increase in the radiative damping of the array.

\section{APPENDIX D: FABRICATION DETAILS}

Figure 7 shows a flow diagram of the fabrication process of our nanoantenna arrays. As a first step, a $2 \times 2$-cm chip was diced from a fused silica wafer. This chip was then cleaned through the use of acetone and isopropyl alcohol (IPA), 


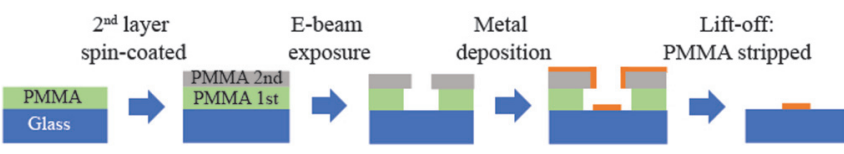

FIG. 7. Flow diagram depicting the fabrication process of our nanoantenna arrays.

followed by blow drying with nitrogen. A 2-wt.-\% PMMA in anisole solution (molecular weight of 450 000) was used as the bottom resist layer. The chip was then spin coated with the bottom resist at $5000 \mathrm{rpm}$ for $60 \mathrm{~s}$ with an acceleration of 300 rps, and then baked at $180{ }^{\circ} \mathrm{C}$ for $30 \mathrm{~min}$, thereby producing a 50-nm-thick bottom resist layer. Similarly, a 2-wt.-\% PMMA in anisole solution (molecular weight of 950 000) was used as the top resist layer. This top layer was spin coated at $7000 \mathrm{rpm}$ for $60 \mathrm{~s}$, with an acceleration of $300 \mathrm{rps}$, thereby producing a 25-nm-thick top resist layer. A nonconductive substrate, such as silica, can result in charge buildup during electron-beam exposure (commonly termed as charging). To avoid this charging effect during electron-beam lithography of our plasmonic nanostructures, espacer, a water-soluble conductive polymer solution, was spin coated at $2500 \mathrm{rpm}$ for $25 \mathrm{~s}$ with an acceleration of $300 \mathrm{rps}$. The plasmonic nanostructures were then patterned using a $30-\mathrm{kV}$ Raith electron-beam lithography system (CRPuO, uOttawa) with a dose of 550 $\mathrm{mC} / \mathrm{cm}^{2}$. After the patterning, the samples were rinsed in deionized water to remove the espacer layer, and postbaked on a hot plate at $80^{\circ} \mathrm{C}$ for $1 \mathrm{~h}$. The resist was then developed for 2 min in 3:1 MIBK-IPA (methyl isobutyl ketone-isopropyl alcohol) mixture at $20^{\circ} \mathrm{C}$, followed by an IPA rinse. With the PMMA bilayer pattern prepared, metallization was carried out by thermal evaporation of $20-\mathrm{nm}$ gold through the use of an Angstrom Nexdep evaporator. As a final step in the fabrication, acetone was used to lift off of the PMMA.
[1] R. H. Dicke, Coherence in spontaneous radiation processes, Phys. Rev. 93, 99 (1954).

[2] N. E. Rehler and J. H. Eberly, Superradiance, Phys. Rev. A 3, 1735 (1971).

[3] R. Bonifacio and L. A. Lugiato, Cooperative radiation processes in two-level systems: Superfluorescence, Phys. Rev. A 11, 1507 (1975).

[4] M. Gross and S. Haroche, Superradiance: An essay on the theory of collective spontaneous emission, Phys. Rep. 93, 301 (1982).

[5] A. V. Andreev, V. I. Emel'yanov, and Y. A. Il'inski, Collective spontaneous emission (Dicke superradiance), Sov. Phys. Usp. 23, 493 (1980).

[6] N. Skribanowitz, I. P. Herman, J. C. MacGillivray, and M. S. Feld, Observation of Dicke Superradiance in Optically Pumped hf Gas, Phys. Rev. Lett. 30, 309 (1973).

[7] M. Gross, C. Fabre, P. Pillet, and S. Haroche, Observation of Near-Infrared Dicke Superradiance on Cascading Transitions in Atomic Sodium, Phys. Rev. Lett. 36, 1035 (1976).

[8] M. Gross, P. Goy, C. Fabre, S. Haroche, and J. M. Raimond, Maser Oscillation and Microwave Superradiance in Small Systems of Rydberg Atoms, Phys. Rev. Lett. 43, 343 (1979).

[9] S. Inouye, A. P. Chikkatur, D. M. Stamper-Kurn, J. Stenger, D. E. Pritchard, and W. Ketterle, Superradiant rayleigh scattering from a Bose-Einstein condensate, Science 285, 571 (1999).

[10] R. G. DeVoe and R. G. Brewer, Observation of Superradiant and Subradiant Spontaneous Emission of two Trapped Ions, Phys. Rev. Lett. 76, 2049 (1996).

[11] J. A. Mlynek, A. A. Abdumalikov, C. Eichler, and A. Wallraff, Observation of Dicke superradiance for two artificial atoms in a cavity with high decay rate, Nat. Commun. 5, 11 (2014).

[12] M. O. Scully, Collective Lamb Shift in Single Photon Dicke Superradiance, Phys. Rev. Lett. 102, 143601 (2009).

[13] A. Angerer, K. Streltsov, T. Astner, S. Putz, H. Sumiya, S. Onoda, J. Isoya, W. J. Munro, K. Nemoto, J. Schmiedmayer, and J. Majer, Superradiant emission from colour centres in diamond, Nature Phys. 14, 1168 (2018).
[14] U. Kreibig and M. Vollmer, Optical Properties of Metal Clusters, Springer series in Materials Science Vol. 25 (SpringerVerlag, Berlin, 1995).

[15] P. K. Jain, W. Huang, and M. El-Sayed, On the universal scaling behavior of the distance decay of plasmon coupling in metal nanoparticle pairs: A plasmon ruler equation, Nano Lett. 7, 2080 (2007).

[16] C. Dahmen, B. Schmidt, and G. von Plessen, Radiation damping in metal nanoparticle pairs, Nano Lett. 7, 318 (2007).

[17] F. Hao, Y. Sonnefraud, P. Van Dorpe, S. A. Maier, N. J. Halas, and P. Nordlander, Symmetry breaking in plasmonic nanocavities: Subradiant LSPR sensing and a tunable Fano resonance, Nano Lett. 8, 3983 (2008).

[18] Y. Sonnefraud, N. Verellen, H. Sobhani, G. A. E Vandenbosch, V. V. Moshchalkov, P. V. Dorpe, P. Nordlander, and S. A. Maier, Experimental realization of subradiant, superradiant, and Fano resonances in ring/disk plasmonic nanocavities, ACS Nano 4, 1664 (2010).

[19] O. L. Muskens, V. Giannini, J. A. Sánchez-Gil, and J. Gómez Rivas, Optical scattering resonances of single and coupled dimer plasmonic nanoantennas, Opt. Express 15, 17736 (2007).

[20] P. Olk, J. Renger, M. T. Wenzel, and L. M. Eng, Distance dependent spectral tuning of two coupled metal nanoparticles, Nano Lett. 8, 1174 (2008).

[21] S. Linden, C. Enkrich, M. Wegener, J. Zhou, T. Koschny, and C. M. Soukoulis, Magnetic response of metamaterials at 100 terahertz, Science 306, 1351 (2004).

[22] C. Rockstuhl, T. Zentgraf, H. Guo, N. Liu, C. Etrich, I. Loa, K. Syassen, J. Kuhl, F. Lederer, and H. Giessen, Resonances of split-ring resonator metamaterials in the near infrared, Appl. Phys. B 84, 219 (2006).

[23] P. Lunnemann, I. Sersic, and A. F. Koenderink, Optical properties of two-dimensional magnetoelectric point scattering lattices, Phys. Rev. B 88, 245109 (2013).

[24] I. Sersic, M. Frimmer, E. Verhagen, and A. F. Koenderink, Electric and Magnetic Dipole Coupling in Near-Infrared SplitRing Metamaterial Arrays, Phys. Rev. Lett. 103, 213902 (2009). 
[25] M. Decker, N. Feth, C. M. Soukoulis, S. Linden, and M. Wegener, Retarded long-range interaction in split-ringresonator square arrays, Phys. Rev. B 84, 085416 (2011).

[26] B. S. Simpkins, J. P. Long, O. J. Glembocki, J. Guo, J. D. Caldwell, and J. C. Owrutsky, Pitch-dependent resonances and near-field coupling in infrared nanoantenna arrays, Opt. Express 20, 27725 (2012).

[27] R. Taubert, R. Ameling, T. Weiss, A. Christ, and H. Giessen, From near-field to far-field coupling in the third dimension: Retarded interaction of particle plasmons, Nano Lett. 11, 4421 (2011).

[28] T. Iida, Control of plasmonic superradiance in metallic nanoparticle assembly by light-induced force and fluctuations, J. Phys. Chem. Lett. 3, 332 (2012).

[29] S. Tokonami, S. Hidaka, K. Nishida, Y. Yamamoto, H. Nakao, and T. Iida, Multipole superradiance from densely assembled metallic nanoparticles, J. Phys. Chem. C 117, 15247 (2013).

[30] M. Tamura, K. Okamoto, K. Tamada, and T. Iida, Stochastic approach to simulation of evaporation-triggered multiple self-assembly of mixed metal nanoparticles and their variable superradiance, Appl. Phys. Lett. 112, 033106 (2018).

[31] K. M. Awan, Fabrication of III-V integrated photonic devices, Ph.D. thesis, Université d'Ottawa, 2018.

[32] L. Novotny and D. Hecht, Principles of Nano-Optics (Cambridge University, Cambridge, England, 2006).

[33] M. Meier, P. F. Liao, and A. Wokaun, Enhanced fields on rough surfaces: Dipolar interactions among particles of sizes exceeding the rayleigh limit, J. Opt. Soc. Am. B 2, 931 (1985).

[34] K. L. Kelly, E. Coronado, L. L. Zhao, and G. C. Schatz, The optical properties of metal nanoparticles: The influence of size, shape, and dielectric environment, J. Phys. Chem. B 107, 668 (2003).

[35] J. E. Sipe and J. Van Kranendonk, Macroscopic electromagnetic theory of resonant dielectrics, Phys. Rev. A 9, 1806 (1974).

[36] D. Tzarouchis and A. Sihvola, Light scattering by a dielectric sphere: Perspectives on the Mie resonances, Appl. Sci. 8, 184 (2018).

[37] P. A. Belov and C. R. Simovski, Homogenization of electromagnetic crystals formed by uniaxial resonant scatterers, Phys. Rev. E 72, 026615 (2005).

[38] S. D. Swiecicki and J. E. Sipe, Periodic Green functions for 2D magneto-electric quadrupolar arrays: Explicitly satisfying the optical theorem, J. Opt. 19, 095006 (2017).
[39] S. Tretyakov, Analytical Modeling in Applied Electromagnetics (Artech House, Boston, MA, 2003).

[40] P. B. Johnson and R. W. Christy, Optical constants of the noble metals, Phys. Rev. B 6, 4370 (1972).

[41] A. A. Svidzinsky, J. T. Chang, and M. O. Scully, Cooperative spontaneous emission of $\mathrm{n}$ atoms: Many-body eigenstates, the effect of virtual lamb shift processes, and analogy with radiation of n classical oscillators, Phys. Rev. A 81, 053821 (2010).

[42] J. H. Eberly, Emission of one photon in an electric dipole transition of one among $\mathrm{n}$ atoms, J. Phys. B 39, S599 (2006).

[43] J. Ruostekoski and J. Javanainen, Quantum field theory of cooperative atom response: Low light intensity, Phys. Rev. A 55, 513 (1997).

[44] H. Freedhoff and J. Van Kranendonk, Theory of coherent resonant absorption and emission at infrared and optical frequencies, Can. J. Phys. 45, 1833 (1967).

[45] J. E. Sipe and J. Van Kranendonk, Energy band models for spatially dispersive dielectric media, Can. J. Phys. 53, 2095 (1975).

[46] B. Luk'yanchuk, N. I. Zheludev, S. A. Maier, N. J. Halas, P. Nordlander, H. Giessen, and C. T. Chong, The Fano resonance in plasmonic nanostructures and metamaterials, Nat. Mater. 9, 707 (2010).

[47] R. Guo, E. Rusak, I. Staude, J. Dominguez, M. Decker, C. Rockstuhl, I. Brener, D. N. Neshev, and Y. S. Kivshar, Multipolar coupling in hybrid metal-dielectric metasurfaces, ACS Photonics 3, 349 (2016).

[48] W. Liu and Y. S. Kivshar, Generalized Kerker effects in nanophotonics and meta-optics, Opt. Express 26, 13085 (2018).

[49] Y. Zhou, I. I. Kravchenko, H. Wang, J. R. Nolen, G. Gu, and J. Valentine, Multilayer noninteracting dielectric metasurfaces for multiwavelength metaoptics, Nano Lett. 18, 7529 (2018).

[50] C. F. Bohren and D. R. Huffman, Absorption and Scattering of Light by Small Particles (Wiley, New York, 2008).

[51] Schott Optical Glass Datasheets, 22 July 2015.

[52] H. Kuwata, H. Tamaru, K. Esumi, and K. Miyano, Resonant light scattering from metal nanoparticles: Practical analysis beyond Rayleigh approximation, Appl. Phys. Lett. 83, 4625 (2003).

[53] S. Zou, N. Janel, and G. C. Schatz, Silver nanoparticle array structures that produce remarkably narrow plasmon lineshapes, J. Chem. Phys. 120, 10871 (2004). 